\title{
International scientists formulate a roadmap for insect conservation and recovery
}

by Harvey, J.A., Heinen, R., Leather, S.R., de Kroon, H. et al.

Copyright, publisher and additional information: .This is the authors' accepted manuscript of a letter. The published version is available via Nature.com and from this

shareable link: $\underline{\text { https://rdcu.be/bZ1zh }}$

Please refer to any applicable terms of use of the publisher.

DOI: https://doi.org/10.1038/s41559-019-1079-8 


\section{International scientists formulate a roadmap for insect conservation and recovery}

Authors: Jeffrey A. Harvey ${ }^{1 *}$, Robin Heinen ${ }^{1}$, Inge Armbrecht ${ }^{2}$, Yves Basset ${ }^{3}$, James H. Baxter-Gilbert $^{4}$, T. Martijn Bezemer ${ }^{1}$, Monika Böhm ${ }^{5}$, Riccardo Bommarco ${ }^{6}$, Paulo A. V. Borges $^{7}$, Pedro Cardoso ${ }^{8}$, Viola Clausnitzer ${ }^{9}$, Tara Cornelisse ${ }^{10}$, Elizabeth E. Crone ${ }^{11}$, Marcel Dicke $^{12}$, Klaas-Douwe B. Dijkstra ${ }^{13}$, Lee Dyer ${ }^{14}$, Jacintha Ellers ${ }^{15}$, Thomas Fartmann ${ }^{16}$, Mathew L. Forister ${ }^{14}$, Michael J. Furlong ${ }^{17}$, Andres Garcia-Aguayo ${ }^{18}$, Justin Gerlach ${ }^{19}$, Rieta Gols $^{13}$, Dave Goulson ${ }^{20}$, Jan-Christian Habel ${ }^{21}$, Nick M. Haddad ${ }^{22}$, Caspar A. Hallmann ${ }^{23}$, Sérgio Henriques ${ }^{5}$, Marie E. Herberstein ${ }^{24}$, Axel Hochkirch ${ }^{25}$, Alice C. Hughes ${ }^{26}$, Sarina Jepsen $^{27}$, T. Hefin Jones ${ }^{28}$, Bora M. Kaydan ${ }^{29}$, David Kleijn ${ }^{30}$, Alexandra-Maria Klein $^{31}$,Tanya Latty ${ }^{32}$, Simon R. Leather ${ }^{33}$, Sara M. Lewis ${ }^{11}$, Bradford C. Lister ${ }^{34}$, John E. Losey $^{35}$, Elizabeth C. Lowe ${ }^{24}$, Craig R. Macadam ${ }^{36}$, James Montoya-Lerma ${ }^{37}$, Christopher D. Nagano $^{10}$, Sophie Ogan ${ }^{25}$, Michael C. Orr ${ }^{38}$, Christina J. Painting ${ }^{39}$, Thai-Hong Pham ${ }^{40}$, Simon G. Potts ${ }^{41}$, Aunu Rauf ${ }^{42}$, Tomas L. Roslin ${ }^{6}$, Michael J. Samways ${ }^{43}$, Francisco SanchezBayo $^{44}$, Sim A. Sar ${ }^{45}$, Cheryl B. Schultz ${ }^{46}$, António O. Soares ${ }^{7}$, Anchana Thancharoen ${ }^{47}$, Teja Tscharntke $^{48}$, Jason M. Tylianakis ${ }^{49}$, Kate D.L. Umbers ${ }^{50}$, Louise E.M. Vet ${ }^{1}$, Marcel E. Visser $^{1}$, Ante Vujic ${ }^{51}$, David L. Wagner ${ }^{52}$, Michiel F. WallisDeVries ${ }^{53}$, Catrin Westphal ${ }^{54}$, Thomas E. White ${ }^{32}$, Vicky L. Wilkins ${ }^{55}$, Paul H. Williams ${ }^{56}$, Kris A.G. Wyckhuys ${ }^{57}$, ZengRong $\mathrm{Zhu}^{58}$, Hans de Kroon ${ }^{23}$

\section{Affiliations:}

${ }^{1}$ Netherlands Institute of Ecology (NIOO-KNAW), Wageningen, The Netherlands.

${ }^{2}$ Universidad del Valle, Departamento de Biología, Cali, Colombia.

${ }^{3}$ ForestGEO, Smithsonian Tropical Research Institute, Panama City, Panama.

${ }^{4}$ Centre for Invasion Biology, Stellenbosch University, Matieland, South Africa.

${ }^{5}$ Institute of Zoology, Zoological Society of London, United Kingdom.

${ }^{6}$ Department of Ecology, Swedish University of Agricultural Sciences, Uppsala, Sweden.

${ }^{7} \mathrm{cE} 3 \mathrm{c}-$ Centre for Ecology, Evolution and Environmental Changes / Azorean Biodiversity, Portugal.

${ }^{8}$ Laboratory for Integrative Biodiversity Research (LIBRe), Finnish Museum of Natural History, University of Helsinki, Finland.

${ }^{9}$ Senckenberg Research Institute, Goerlitz, Germany.

${ }^{10}$ Center for Biological Diversity, Portland, Oregon, USA

${ }^{11}$ Department of Biology, Tufts University, Medford MA, USA.

${ }^{12}$ Laboratory of Entomology, Wageningen University, Wageningen, The Netherlands.

${ }^{13}$ IUCN SSC Freshwater Conservation Committee, Naturalis Biodiversity Center, Leiden, The Netherlands.

${ }^{14}$ Biology Department, University of Nevada, Reno, USA.

${ }^{15}$ Department of Ecological Sciences, VU University, Amsterdam, The Netherlands. 
${ }^{16}$ Osnabrück University, Department of Biodiversity and Landscape Ecology, Osnabrück, Germany.

${ }^{17}$ School of Biological Sciences, The University of Queensland, St Lucia 4072, Queensland, Australia.

${ }^{18}$ Estacion de Biología Chamela, Instituto de Biología, Chamela, Jal., Mexico.

${ }^{19}$ IUCN SSC Terrestrial Invertebrate Red List Authority, Cambridge, United Kingdom.

${ }^{20}$ University of Sussex, School of Life Sciences, United Kingdom.

${ }^{21}$ Evolutionary Zoology, University of Salzburg, Department of Biosciences, Salzburg, Austria.

${ }^{22}$ Kellogg Biological Station and Department of Integrative Biology, Michigan State University, Hickory Corners MI, USA.

${ }^{23}$ Institute for Water and Wetland Research, Radboud University, Nijmegen, The Netherlands.

${ }^{24}$ Department of Biological Sciences, Macquarie University 2109 NSW Sydney, Australia.

${ }^{25}$ Trier University, Department of Biogeography, Trier, Germany.

${ }^{26}$ Centre for Integrative Conservation, Xishuangbanna Tropical Botanical Garden, Chinese Academy of Sciences, Menglun, Yunnan, China.

${ }^{27}$ The Xerces Society for Invertebrate Conservation, Portland, Oregon, USA.

${ }^{28}$ School of Biosciences, Cardiff University, Cardiff, United Kingdom.

${ }^{29}$ Çukurova University, Biotechnology Application and Research Centre, Balcalı, Adana, Turkey.

${ }^{30}$ Plant Ecology and Nature Conservation Group, Wageningen University, Wageningen, The Netherlands.

${ }^{31}$ Albert Ludwigs University of Freiburg, Germany.

${ }^{32}$ School of Life and Environmental Science, Sydney Institute of Agriculture, University of Sydney, NSW, Australia.

${ }^{33}$ Crop \& Environment Science, Harper Adams University, Edgmond, Newport, United Kingdom.

${ }^{34}$ Department of Biological Sciences, Rensselaer Polytechnic Institute, Troy, New York, USA.

${ }^{35}$ Entomology Department, Cornell Univeristy, Ithaca, New York, USA.

${ }^{36}$ Buglife - The Invertebrate Conservation Trust, United Kingdom.

${ }^{37}$ Universidad del Valle, Departamento de Biología, Cali, Colombia.

${ }^{38}$ Key Laboratory for Zoological Systematics and Evolution, Institute of Zoology, Chinese Academy of Sciences, Beijing, China.

${ }^{39}$ School of Science, University of Waikato, Hamilton, New Zealand.

${ }^{40}$ Vietnam National Museum of Nature \& Graduate School of Science and Technology, Vietnam Academy of Science and Technology, Hanoi, Vietnam.

${ }^{41}$ Centre for Agri-Environmental Research, School of Agriculture, Policy and Development, Reading University, United Kingdom. 



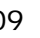
10

${ }^{42}$ Department of Plant Protection, IPB University, Bogor, Indonesia.

${ }^{43}$ Dept. of Conservation Ecology and Entomology, Stellenbosch University, Matieland, South Africa.

${ }^{44}$ Department of Environment and Energy, Canberra, Australia.

${ }^{45}$ National Agricultural Research Institute,Lae Papua New Guinea.

${ }^{46}$ School of Biological Sciences, Washington State University, Vancouver, USA.

${ }^{47}$ Department of Entomology, Faculty of Agriculture, Kasetsart University, Thailand.

${ }^{48}$ Agroecology, Department of Crop Sciences, University of Göttingen, Göttingen, Germany.

${ }^{49}$ Bio-protection Centre, School of Biological Sciences, University of Canterbury, Christchurch, New Zealand.

${ }^{50}$ School of Science and Health, Western Sydney University, Australia.

${ }^{51}$ Faculty of Sciences, Department of Biology and Ecology, University of Novi Sad, Novi Sad, Serbia.

${ }^{52}$ Ecology and Evolutionary Biology, Unversity of Connecticut, Storrs CT, USA.

${ }^{53}$ De Vlinderstichting (Dutch Butterfly Conservation) \& Plant Ecology and Nature Conservation Group, Wageningen University, Wageningen, The Netherlands.

${ }^{54}$ Functional Agrobiodiversity, Department of Crop Sciences, University of Göttingen, Germany.

${ }^{55}$ IUCN SSC Mid Atlantic Island Invertebrate Specialist Group, IUCN, Cambridge, United Kingdom

${ }^{56}$ Natural History Museum, London, United Kingdom.

${ }^{57}$ Chrysalis Consulting, Hanoi, Vietnam.

${ }^{58}$ Zhejiang Provinvial Key Laboratory of Crop Insect Pests and Diseases, Hangzhou, Zhejiang, China.

*Correspondence to Jeffrey A. Harvey, j.harvey@nioo.knaw.nl Droevendaalsesteeg 10, 6708PB, Wageningen, The Netherlands, Orcid number: 0000-0002-4227-7935 tor 
A growing number of studies are providing evidence that a suite of anthropogenic stressors - habitat loss and fragmentation, pollution, invasive species, climate change and overharvesting - are seriously reducing insect and other invertebrate abundance, diversity and biomass across the biosphere ${ }^{1-9}$. These declines affect all functional groups: herbivores, detritivores, parasitoids, predators, and pollinators. Insects are vitally important in a wide range of ecosystem services ${ }^{10}$ of which some are vitally important for food production and security (e.g. pollination and pest control) $)^{11}$. There is now a strong scientific consensus that the decline of insects, other arthropods and biodiversity as a whole, is a very real and serious threat that society must urgently address ${ }^{12-14}$. In response to the increasing public awareness of the problem, the German government is committing funds to combat and reverse declining insect numbers ${ }^{14}$. This funding should act as a clarion call to other nations across the worldespecially wealthier ones - to follow suit and to respond proactively to the crisis by addressing the known and suspected threats and implementing solutions.

We hereby propose a global 'roadmap' for insect conservation and recovery (Fig. 1a). This entails the immediate implementation of several 'no-regret' measures (Fig. 1a,b) that will act to slow or stop insect declines. Among the initiatives we encourage are the following immediate measures:

Taking aggressive steps to reduce greenhouse gas emissions; reversing recent trends in agricultural intensification including reduced application of synthetic pesticides and fertilizers and pursuing their replacement with agro-ecological measures; promoting the diversification and maintenance of locally-adapted land use techniques; increasing landscape heterogeneity through the maintenance of natural areas within the landscape matrix and ensuring the retention and creation of microhabitats within habitats which may be increasingly important for insects during extreme climatic events such as droughts or heatwaves; reducing identified local threats such as light, water or noise pollution, invasive species etc.; prioritizing the 
import of goods that are not produced at the cost of healthy, species-rich ecosystems; designing and deploying policies (e.g. subsidies, taxation) to induce the innovation and adoption of insect-friendly technologies; enforcing stricter measures to reduce the introduction of alien species, and prioritizing nature-based tactics for their (long-term) mitigation; compiling and implementing conservation strategies for species that are vulnerable, threatened or endangered; funding educational and outreach programs, including those tailored to the needs of the wider public, farmers, land managers, decision makers and conservation professionals; enhancing 'citizen science' or 'community science' as a way of obtaining more data on insect diversity and abundance as well as engaging the public, especially in areas where academic or professional infrastructure is lacking; devising and deploying measures across agricultural \& food value chains that favor insect-friendly farming, including tracking, labeling, certification and insurance schemes or outcome-based incentives that facilitate behavioral changes, and investing in capacity-building to create a new generation of insect conservationists and providing knowledge and skills to existing professionals (particularly in developing countries).

To better understand changes in insect abundance and diversity, research should aim to prioritize the following areas:

Quantifying temporal trends in insect abundance, diversity and biomass by extracting long-term data sets from existing insect collections to inform new censuses; exploring the relative contributions of different anthropogenic stressors causing insect declines within and across different taxa; initiating long-term studies comparing insect abundance and diversity in different habitats and ecosystems along a management-intensity gradient and at the intersection of agricultural and natural habitats; designing and validating insect-friendly techniques that are effective, locally-relevant and economically-sound in agriculture, managed habitats and urban environments; promoting and applying standardized monitoring protocols 
globally and establishing long-term monitoring plots or sites based on such protocols, as well as increasing support for existing monitoring efforts; establishing an international governing body under the auspices of existing bodies (e.g., UNEP or IUCN) that is accountable for documenting and monitoring the effects of proposed solutions on insect biodiversity in the longer term; launching public-private partnerships and sustainable financing initiatives with the aim of restoring, protecting and creating new vital insect habitats as well as managing key threats; increasing exploration and research to improve biodiversity assessments, with a focus on regional capacity building in understudied and neglected areas, and performing large-scale assessments of the conservation status of insect groups to help define priority species, areas and issues.

Most importantly, we should not wait to act until we have addressed every key knowledge gap. We currently have enough information on some key causes of insect decline to formulate no-regret solutions whilst more data are compiled for lesser known taxa and regions and long term data are aggregated and assessed. Implementation should be accompanied by research that examines impacts, the results of which can be used to modify and improve the implementation of effective measures. Furthermore, such a 'learning-bydoing' approach ensures that these conservation strategies are robust to newly emerging pressures and threats. We must act now.

\section{References}

1. Biesmeijer JC, et al. Science 313, 351-354 (2006).

2. Dirzo R, Young HS, Galetti M, Ceballos G, Isaac NJB, Collen B. Science 345, 401-406 (2014). 
3. Hallmann CA, et al. PLoS One 12, e0185809 (2017).

188

4. Leather S. Ann Appl Biol 172, 1-3 (2017).

190

191

5. Lister BC, Garcia A. Proc Natl Acad Sci USA 115, E10397-E10406 (2018).

192

6. Sánchez-Bayo F, Wyckhuys KAG. Biol Conserv 232, 8-27 (2019).

194

195

7. van Strien AJ, van Swaay CAM, van Strien-van Liempt WTFH, Poot MJM, WallisDeVries MF. Biol Conserv 234, 116-122 (2019).

197

201

202

10. Messer E, Cohen MJ. Food Cult Soc 10, 297-315 (2007).

203

204

11. Hochkirch A. Nature 539, 141-141 (2016).

205

13. Basset Y, Lamarre GPA. Science 364, 1230-1231 (2019).

209

210

211

8. Siebold S. et a. Nature 574, 671-674 (2019).

9. Losey JE, Vaughan M. Bioscience 56, 311-323 (2006).

12. Pina S, Hochkirch A. Science 356, 1131-1131 (2017).

14. Vogel G. Science 364, 519-519 (2019). 


\section{$212 \quad$ Figure legend}

213 Figure 1. Road map to insect conservation and recovery calling for action at short-,

214 intermediate- and long-term timescales. No-regret measures for immediate utilization in

215 insect conservation refer to actions that should be implemented as soon as possible. These

216 solutions will be beneficial to society and biodiversity even if the direct effects on insects are

217 not known as of yet (i.e., no-regret solutions). This encompasses utilization of insect-friendly

218 techniques that are effective, locally relevant and economically sound, e.g., in farming, habitat

219 management and urban development.

220

221 Competing Interests

222 The authors declare no competing interests. 